\title{
Atomic Structure and Binding of Carbon Atoms
}

\section{Mubarak Ali}

Department of Physics, COMSATS University Islamabad, Park Road, Islamabad45550, Pakistan, E-mail: mubarak74@mail.com

Abstract - Many studies discuss carbon-based materials because of the versatility of the carbon element. They present different sorts of understandings fairly at convincing and compelling levels. A gas-state carbon atom converts into its various states depending on the conditions of processing. The electron transfer mechanism in the gas-state carbon atom is responsible for its conversion to various states, namely, graphite, nanotube, fullerene, diamond, lonsdaleite and graphene. The shape of energy responsible to transfer electron from the sides (east- and westpoles) of its atom is like parabola. That energy is linked to states (from filled state to nearby unfilled state) where exerted force to relevant poles of transferring electron is remained neutral. So, the mechanism of originating different states from a gaseous carbon atom is under the involvement of energy at first, which is not the case for atoms executing their confined inter-state electron-dynamics where force is involved at first. Structure evolved in graphite-, nanotubes- and fullerene-states have respectively one-dimensional, two-dimensional and four-dimensional atoms. Moreover, the associated energy curve is a parabola, indicating the transfer of electrons under neutral exertion of forces to their relevant poles. The graphite structure under only attained-dynamics of atoms is also developed but in twodimension. Here, binding energy between graphitic carbon atoms is engaged under the influence of a small difference available between their involved forces along opposite poles. Structural evolution in diamond, lonsdaleite and graphene atoms involve potential energy of electrons required to undertake infinitesimal displacements under orientationally-controlled exerting forces to their relevant poles. In this study, the growth of diamond is found to be south to ground where atoms bound ground to south. Thus, diamond atoms merge for a tetra-electron ground to south topological structure. Lonsdaleite atoms merge for a bi-electron ground to justsouth topological structure. The growth of graphene was just-north to ground; however, the binding of atoms was ground to just-north showing tetra-electrons 
ground to just-north topological structure. Glassy carbon exhibits layered-topological structure which successively binds tri-layers of gas-, graphite- and lonsdaleite-state atoms in repetitive manner. Orientating pair of electrons of each atom of below gas layer and above lonsdaleite layer enter from the rear side and front side respectively to undertake another clamping of unfilled energy knots belonging to each atom of intermediate graphitic layer. Different carbon atoms develop amorphous structures when they bind under frustrating amalgamation. Hardness of carbon-based materials was also sketched in the light of force-energy behaviors of different state carbon atoms. Here, structure evolution in each carbon state atom explores its own science. Keywords: Carbon atomic structure; Allotropes; Electron-dynamics; Energy; Neutral force; Non-conservative force; Atomic binding

\subsection{Introduction}

Developing materials of selective size and shape, and investigating their characteristics for various applications, solicit new sort of approaches and observations. The forces appearing at electron-levels involve the process of structural modification, which involve energy changes and vice versa. The engagement of neutral forces or non-conservative forces at electron-levels is because of the execution of their dynamics under partial-confinement or nonconfinement of inter-state where energy should involve first. The atoms of various carbon allotropes are appeared to be the candidates of such forces as the available filled and unfilled states are just near to the centers of their respective-state atoms. The force and energy of the atoms are to be considered in partially conservative mode in the former case, and in the non-conservative (frictional) mode entirely in the latter case. Engaging (or involving) energy to involve (or engage) force for evolving structure of different format solid-natured atoms is to be considered as per their builtin gauge of electron-dynamics. Here, the word "involve" refers to an action of energy or force for "instant time", whereas, the word "engage" refers to an action of energy or force for "eternal period". When carbon atoms of gas-state are converted into its certain state eligible to evolve structure, the involvement of energy first rather than the force is expected. In each carbon atom, electrons of outer ring should be considered to execute dynamics where they are close enough to electrons belonging to zeroth ring. A zeroth ring is related to center of an atom, which constitutes four electrons. So, electrons of zeroth ring don't permit force to appear for their relevant 
poles. They should not undertake their transferring mechanism as for the case of electrons of outer ring (first ring) of a carbon atom. In a suitable carbon, electrons of the outer ring obey transfer mechanism because of the uncovered-sides of filled states and unfilled states. But, due to their limitation to be too close to center of their atom, they should involve the energy first for transferring to suitable unfilled states. However, when conservative forces are exerted on electron of neutral state silicon atom, an uninterrupted execution of electron-dynamics results into wave-like heat energy [1]. This indicates that the built-in gauge of electron-dynamics in the case of carbon atom is different as compared to silicon atom, despite the fact that same number of filled and unfilled states are available in their outer ring. But the distance of each electron of the outer ring from the center in carbon atom is different compared to silicon atom [2]. So, silicon atom executes confined inter-state electrondynamics instead of non-confined (or partially confined) inter-state electrondynamics. The atoms at different levels involve conservative forces, where confined inter-state electron-dynamics are executed. This evolves the structures in different manner [3]. This approach based on the multidisciplinary point of view may originate understandings of a different atomic nature and behavior which could be very different from that available in the existing literature.

It is also important to know that atoms belonging to any element don't ionize [4]. Understanding the mechanism of evolving structure based on the different carbon states rely on same chemistry at the input end. Various spectroscopic analyses of a 'tiny grains carbon film' give peaks at different positions indicating different carbon states/structures [5]. In addition, depending on the conditions and the techniques involved, gas carbon atom-source works for the development of different morphology and structure of grains and crystallites [6]. Moreover, different morphology of grains and crystallites (particles) is observed at different chamber pressure identifying the role of arresting energies near to/at substrate with different rate for each resident chamber pressure [7]. Moreover, the deposition of graphite and diamond in distinctive manner at single substrate is due to the different set inter-wire distance of dissociating gases [8].

Carbon atoms having different states are known as allotropes, i.e., starting from the gas-state carbon atom to graphite-state, then, diamond, lonsdaleite carbon, fullerene followed by nanotube-state, glass carbon, and recently, the graphene. Several studies on carbon-based materials are available in the literature explaining 
the conditions of deposition and their effects in the form of morphology, growth rate, quality and application, etc.

It is necessary to understand the dynamics of development of tiny particles prior to assembling them into large-sized particles [9]. Agglomerations of colloidal matter envisage atoms and molecules to deal them as materials for tomorrow [10]. Developing of different-feature tiny particles have been discussed elsewhere [11]. The developing mechanism of tiny-shaped particles under certain concentration of gold precursor has been discussed [12]. Under identical process parameters, the nature of precursor directs tiny-shaped particles following by the development of their large-shaped particles where role of the required atomic nature was also in focus [13]. Different tiny particles following by large-sized particles were developed under the application of nano-energy, while varying the bipolar and unipolar pulses times [14]. Developing large-sized particles shows very high development rate [15]. Developing monolayer tiny-shaped particle under the application of nano-energy was discussed where atoms of one-dimensional arrays are converted into structures of smooth elements [16]. Certain nature atoms of tiny-sized particles undertake different behaviors resulting into work as either effective or defective nanomedicine [17]. Gold particles of unprecedented shapes have been developed under tailored conditions of processing solution [18]. Hard coating of certain gas- and solid-natured atoms developed because of their established transitional force-energy behaviors [19].

Atoms of different elements and then structures are recognized by their physical behavior. A carbon atom shows several physical natures even though it exhibits a distinctive chemical nature. Different carbon-based materials possess atoms of same recognized chemistry but indicate a very different behavior with respect to each other [5-8]. This indicates that the transition of electrons within designated states to nearby unfilled state (within the same ring) change the chemical nature of atom resulting into a new state of physical phenomenon. In gas- and solid-natured atoms of suitable elements, transitions of electrons can't cross the north-pole or south-pole of their atom, but they do cross their own projected north-south poles to develop liquid transition state [2]. It is also observed that the force entering (north-pole) and leaving the ground surface (south-pole) is different as compared to force at/near ground surface (east-west poles) [20]. Thus, the available option for transferring electron of filled state to unfilled state in all suitable atoms is left at left-side or right-side of their 
atoms. So, the available option for transition of electrons in gaseous or solid atoms (belonging to the suitable elements) is only within the clamped energy knots where they undergo established transition states namely, recovery, neutral, recrystallization and liquid states depending on the rate of their infinitesimal displacements. The center of each atom is related to null exerting forces of different poles as it is declared as the common point of inter-crossed overt photons having no mass of the electrons [2]. When the ground point of an atom is above the ground surface, as in the gas state, the dominating force is considered to be based on the space-format. When the ground point of an atom is below ground surface, as in solid state, the dominating force is considered to be based on the grounded-format. When the ground point of an atom is at average-leveled ground surface, as in partial solids, the dominating force is considered because of surface-format. Evolution of different dimension structures in atoms of nearly (semi) solid structures, solid behavior and highly solid behavior at just above ground surface, at ground surface and at below ground surface, respectively, envisage different format of exerting forces at electronlevels [3].

A recent study shows transformation of graphene film into a diamond-like film where the elastic deformations and chemical natures were changed [21]. Wu et al. [22] reviewed the developments in Raman spectroscopy of graphene-based materials from both fundamental research and practical perspectives. Uniform carbon nanofibers are grown by vapor deposition method without involving the catalyst [23]. Different applications related to graphene hybrids were reviewed recently in a study [24]. Nitrogen incorporated carbon dots were used to modify a glassy carbon electrode [25]. A novel energy dissipation system was investigated by combining the carbon nanotube and buckyballs [26]. Different carbon allotropes were studied for the dehydrogenation temperature in their comparison [27]. A precise positioning of the vacancies within the diamond crystal was studied by Chen et al. [28]. Liu et al. [29] presented an efficient strategy of electrochemical activation to fabricate the graphite-graphene Janus architecture. Repeated large-area doped nano-crystalline diamond layers prepared under optimized conditions of microwavebased vapor deposition system [30]. Cheng and Zong [31] observed structural evolution of damaged carbon atoms for deeper surface layer. Maruyama and Okada [32] investigated geometric, electronic and magnetic structures of a two-dimensional network of carbon atoms. Narjabadifam et al. [33] studied both elastic and failure 
properties of carbon nanocones through the application of molecular dynamics simulation.

Binding of atoms in different state shows that the carbon atoms remains challenging since the discovery, and only partial information on evolution of graphite structure is available. In addition, atom to atom binding of carbon in diamond remains challenging as, on one side, a large crystallite (particle) is growing and, on the other, a single atom of diamond is being deposited on it for further growth. The origin of structure in other carbon states as well as evolution of structure comprising layers of certain state carbon atoms in a repeated order remains challenging. In the present work, atomic structure of different carbon states is pinpointed along with structure evolution of all possible carbon states. This study describes the science of originating different carbon states and their structure evolution.

\subsection{Results and discussion}

The lattice of a carbon atom; Figure 1 (a) shows four unfilled states (energy knots) at the center which are related to the zeroth ring, whereas, eight unfilled states (energy knots) around the zeroth ring are related to outer ring (first ring). Each energy knot is formed by the precise inter-crossing of the overt-photons having certain length, where the trough of one is in-front of the crest resulting into freeze (jam) the element of their force. Two pairs of overt-photons having wavelength of current when intercrossed by keeping their common-centre, eight states of electrons are formed, which are related to the eight hollow regions where the element of force remains intact along with the energy. Because, a photon constitutes both the element of force and energy in its formation (generation). Those two pairs of photons (having characteristic of current) inter-crossed (at the same center) along north-south axes compress two states (energy knots) of their opposite sides by means of already inter-crossed double pair along east-west poles. This results into the hollow regions for only four states of electrons as shown in Figure 1 (a). Pairs of certain length overt-photons inter-crossed to form the states of clamping energy knots of twelve electrons under their common-centre. Among these twelve states, central four are related to zeroth ring while the outer ring of eight sites form the first ring (where four remained filled and four remained unfilled). This ordering provides the option to originate six different state behaviors of a carbon atom in addition to the gas-state. In Figure 1 (b), a gas-state carbon atom is shown, while remaining different states of a 
carbon atom are shown in Figure 1 (c-h), where the positions of electrons belonging to the outer ring are changed accordingly; in (c) graphite-state, (d) nanotube-state, (e) fullerene-state, (f) diamond-state, (g) lonsdaleite-state and (h) graphene-state carbon atoms are shown. For each carbon atom, the central four electrons form the zeroth ring, which is termed as nucleus and is a helium atom [2].

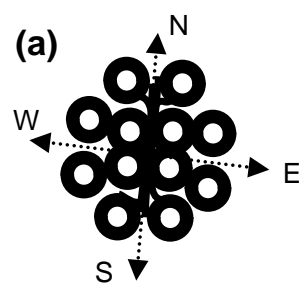

(e)

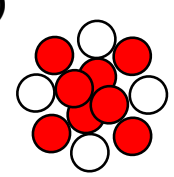

(b)

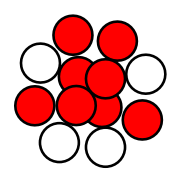

(f)

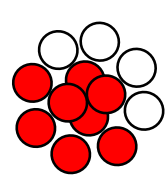

(c)

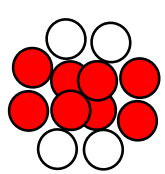

(g)

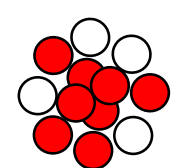

(d)

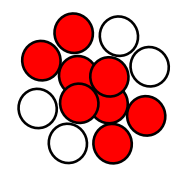

(h)

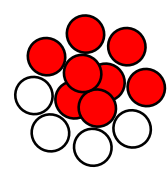

Figure 1: (a) lattice of a carbon atom, atomic structure of carbon atom when in (b) gas-state, (c) graphite-state, (d) nanotube-state, (e) fullerene-state, (f) diamond-state, (g) lonsdaleite-state and (h) graphene-state; filled red colored circles indicate filled states, filled white colored circles indicate unfilled states and black colored rings indicate clamping energy knots to states; drawn in estimation

A gas-state carbon atom is processed to transfer two electrons of filled state to nearby unfilled state; one from the right-side and one from the left-side for one state migration. As a result, energy having shape of built-in gauge of the electrondynamics is involved where exerting forces of relevant poles to those electrons remain neutral. Thus, a transferring electron obeys the exact trajectory of bound energy to energy knots of filled and unfilled states. Each binding energy plot will have electron for transfer, one from the east-side and one from the west-side of the atom, enabling conversion of gas-state carbon atom into graphite-state. In transferring all four electrons of outer ring to unfilled states available below to eastwest poles (central line) in gas-state carbon atom, three pairs of energy plot have shape-like parabola involved, which results to convert it into a diamond-state carbon atom. The applied forces on relevant poles for transferring electrons to each dedicated state of their atom remains neutral.

When gas-state carbon atom converts into graphite, it is under the availability of energy shape-like parabola where certain electron of left-side and right-side are transferred by the neutral behaviors of forces applied for them. The exerted forces are related to space-format and surface-format, which are kept neutral at the instant of transferring electrons because of the blockage of electrons belonging to zeroth 
ring of that carbon atom. However, transferring requires electrons of graphitic carbon atom for lonsdaleite-state carbon atom. So, an energy plot having parabola like shape along west to south and an energy curve-like parabola along east to south are involved. Here, the exerting forces for those electrons remained under the neutral behavior. But, exerted forces for transferring of electrons are related to surfaceformat and grounded-format. In conversion of lonsdaleite-state atom from graphitestate, only two electrons are transferred to dedicated states under typical energies. On conversion of diamond-state atom from lonsdaleite, two electrons are transferred further to dedicated states under involved energies. At that instant, ground point of the diamond-state atom becomes further below to ground surface as compared to lonsdaleite-state atom, as it possesses ground point at just below the ground surface. Transfer of all (four) electrons of outer ring towards south-pole in a carbon atom (two from the left-side of south-pole and two from the right-side of the southpole) results into ground point of the carbon atom to become fully grounded. This gives the diamond-state carbon atom. Thus, the transferred electron undertakes the maximum potential energy, which is being maintained under the exertion of orientating gravitational force to the maximum extent where clamped energy knots to electrons possess the maximum expansion also.

Due to the very small distance of outer ring from the center of carbon atom, exerting forces of east and south poles, east and north poles, west and south poles and west and north poles, for transferring a filled state electron to nearby unfilled state, in each case, becomes almost equal. Thus, energy shape-like parabola regulates it for the entire trajectory forming between filled to nearby unfilled state. So, the relevant forces influence under the neutral behavior while transferring electrons of opposite sides in carbon atom and under the involved set of typical energies. Therefore, the carbon atom originates a new state behavior under the maintenance of equilibrium. Under the maintenance of equilibrium, transferring electrons of outer ring in graphitic carbon atom converts it into fullerene-state carbon atom. A gas-state carbon atom converts into nanotube-state carbon atom. But, a fullerene-state atom can be converted directly into nanotube-state under the supply of two unit-energy shape-like parabola. A graphitic carbon atom is converted into diamond-state, lonsdaleite-state and graphene-state carbon atom, one by one, under the supply of different number unit-energy shape-like parabola, where the exerting forces to electrons do not disturb them to follow the trajectory as they remain neutral for all 
poles of an electron. Energy knots forming the unfilled and filled states of the carbon lattice expand or contract to different extent depending on the position of electrons belonging to the outer ring.

An occupied or unoccupied position of electron in the atom is termed as 'state'. Based on newly occupied state of the electron, a new allotrope of its carbon atom is also termed as 'state' but, in this case, it is the atomic state instead of electron state. Depending on the electrons-attained positions in their carbon atom, the contraction and expansion of clamped energy knots are adjusted accordingly and, then, relatively to the neighboring ones.

In Figure 2 (a), binding of graphitic carbon atoms is shown; where one amalgamated atom is already in the graphite state (atom A) and the other (atom B) is in the transition to attain the graphite state. At that instant, energies involved for transferring the electrons of filled states to the ones for attaining graphite-state of that carbon atom is also utilized for binding. Thus, graphite-state carbon atoms are bound adjacently along the same axis. However, the energy is absorbed (or linked between states) prior to transfer of an electron where it follows its trajectory having shape-like parabola to attain the graphite-state where role of the engaged forces (in both space-format and surface-format) remained impartial (unbiased/neutral). Thus, the atom $B$ is bound to atom $A$ while converting to graphite-state. Binding of atom $B$ to atom $A$ involves typical energies having plot shape-like parabola and results in the graphite structure by the repetition of the same scheme. In the binding of carbon atoms in graphitic carbon atoms, the energy involved is also protected by the neutral behavior of applied forces as the shape of typical energy and its connections between nearby relevant energy knots do not affect. The applied neutral behavior of forces in binding atoms in graphite remain along the same axis as shown in Figure 2 (a), thus, developing graphite structure in one dimension. In developing graphite structure under the execution of electron-dynamics of atoms, their binding remains along the single direction of $\mathrm{X}$-axis. Under the execution of electron-dynamics, this one-dimensional structure evolution in graphitic carbon atoms can be in the single direction of opposite-side of X-axis. Atoms of such one-dimensional arrays while evolving their tiny grains, elongate under the exertion of forces in surface-format to convert them into structures of smooth elements [5]. 

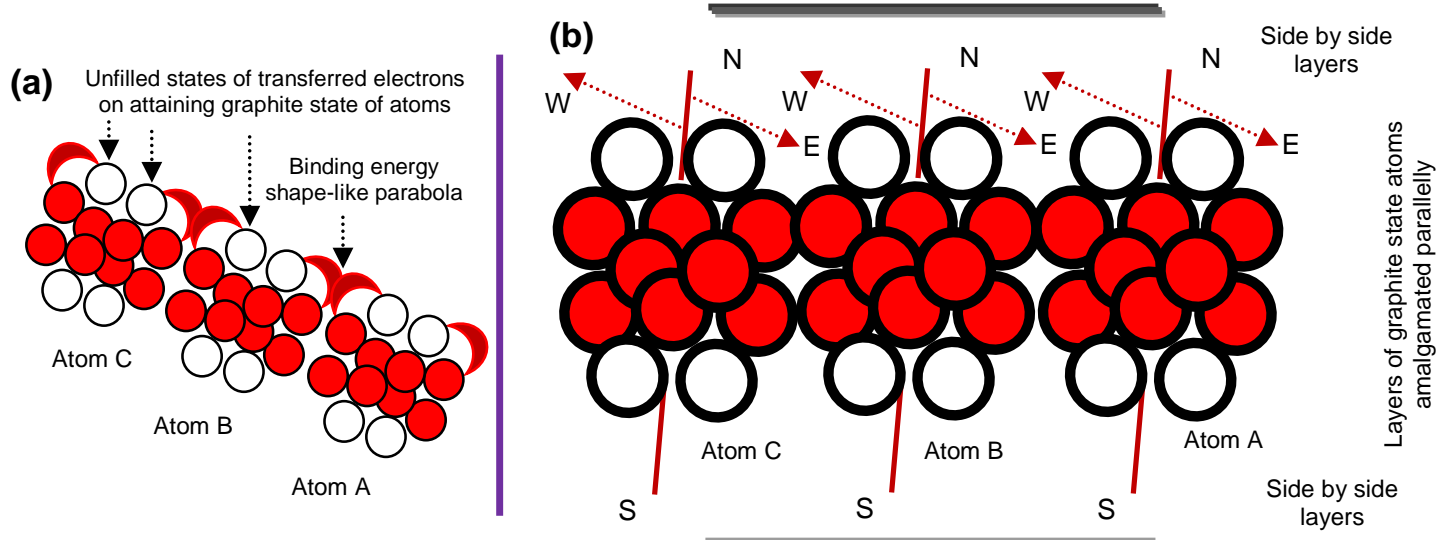

Figure 2: Structure evolution in carbon atoms of graphite-state (a) when executing electrondynamics under suitable amalgamation, they evolve a one-dimensional structure and where (b) amalgamated atoms engaged forces only under the attained dynamics, they evolve a twodimensional structure

When atoms amalgamated under preserving their state of graphite, they only bind under the attained dynamics to evolve structure without the execution of electron-dynamics. At this stage, the evolving graphite structure becomes twodimensional. The typical parabola like energy curve remains no more involved in binding graphitic carbon atoms. Based on the slight difference in exerting east-west forces at point of amalgamated two graphite-state atoms to central one, they remain bind only under attained dynamics, Figure 2 (b). When identical layers of graphitestate atoms are developed side by side (parallelly) to that layer, upward-side and downward-side (or only for one side), it undertakes two dimensions as the force differs along the opposite poles of atoms while forming their layers. Even though existing forces of opposite poles don't work for an appreciable difference to allow binding of graphite-state carbon atoms, they also don't allow atoms to go away from each other. Under the opposite poles' forces of east-west, graphite-state carbon atoms adjust along both directions of $X$-axis where they amalgamate adjacently.

When the surface of evolving graphite structure is not flat at electron-level, the influences of exerting north-south forces are also included where developing structure of graphitic carbon atoms is related to amorphous graphite structure. The evolution of amorphous structures may be considered in the case of other states of carbon atoms where atoms can distort evolving structure of a certain state carbon atom. Uneven surfaces for developing the first layer utilize the forces of north-south poles under non-uniform distribution, resulting in maintaining developed amorphous graphite structure. 
A nanotube-state carbon atom, converted from the fullerene-state carbon atom prior for assembling, is under the neutral forces while transferring electrons. Therefore, transferring electrons for each unfilled state is also under the involved energy shape-like parabola. Here, the execution of dynamics of electron is neither under non-conservative force, nor under conservative force, but rather under partial conservative force as the energy curve shape-like parabola. Carbon atom of nanotube-state evolves structure based on the involvement of binding energy for atoms attaining the identical state at the instance of amalgamating, Figure 3 (a). Here, a fullerene-state carbon atom converts into a nanotube-state carbon atom on transferring electron to nearby unfilled state for each opposite quadrant. Atoms of such carbon state bind under the neutral behavior of exerting forces to electron in surface-format and space-format for one quadrant, and exerting forces to electron in surface-format and grounded-format for the opposite quadrant. In the case of structure of nanotube evolution, atoms of nanotube-state execute their electrondynamics in two opposite quadrants, where neutral behavior of forces are exerted also while transferring electron to the dedicated state. The energy shape-like parabola is involved to transfer electron of a particular state, from south-side and north-side in the opposite quadrants of atom resulting in binding amalgamating atoms at both sides, Figure 3 (a). The binding of atoms in nanotube structure has two opposite quadrants, in either way, the evolution of the structure is related to two dimensions, but the overall shape of nanotube appears in one-dimensional shape, which is shown for two options in Figure 3 (a).

A carbon atom attains fullerene-state by the transfer of electron at each dedicated state of pole while engaging the energy shape-like parabola for all four quadrants, where transferring electron of each quadrant engages neutral behavior of exerting forces of relevant poles. Here, a contribution of applied neutral force in space-format for two quadrants along with surface-format, and a contribution of exerting neutral force in grounded-format for two quadrants along with surfaceformat is to be considered. A characteristic energy shape-like parabola while binding of identical state atoms at point of executing electron-dynamics is shown in Figure 3 (b); where the evolution of fullerene structure in two different ways is shown. This indicates that the structural evolution in fullerene-state carbon atoms is fourdimensional. Binding of fullerene-state carbon atoms to evolve fullerene structure for all four quadrants of nearly plane surface forming angle either at nearly mid of each 
quadrant or at nearly along north-south poles and east-west poles. A fullerene-state carbon atom converts from the nanotube-state carbon atom before assembling, where exerting forces of relevant poles at the time of transferring electrons remain neutral and only the involved energy transferred them to dedicated states. In the fullerene-state carbon atom, upper-sided transferred electrons engage the neutral forces in space-format and surface-format, while lower-sided transferred electrons engage the neutral exertion of forces in grounded-format and surface-format.

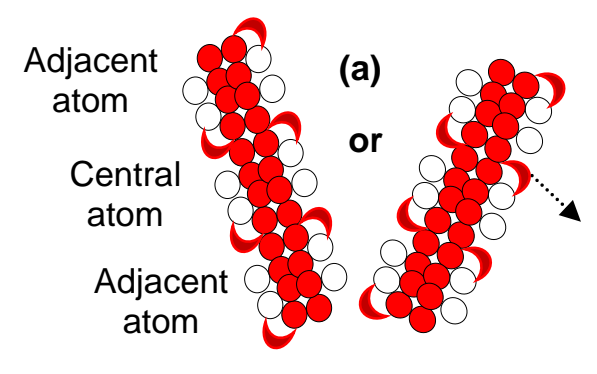

(b)

Figure 3: (a) nanotube structure - a two-dimensional structure where the involved energy shapelike parabola in opposite quadrants of targeted atom binds amalgamated atoms at left-side and rightside and (b) fullerene (buckyballs) - a four-dimensional structure where the energy shape-like parabola involved in each quadrant of targeted atom binds four amalgamated atoms

A lonsdaleite-state carbon atom having ground point just below ground surface is shown in Figure 4 (a). It approaches to bind to the diamond-state atom once it is converted into a diamond-state atom. A diamond-state atom, which has already attained ground point at sufficiently below surface, is also shown in Figure 4 (a). The expected binding point of atoms, when both are binding in diamond state, is also shown in the Figure 4 (a). In the nucleation of synthetic diamond, a deposited atom is at highly-heated scratched-seeded surface of solid which doesn't allow it further to attempt gravitation behavior of electrons. This is because of having their maximum potential energy under orientationally-controlled exerting forces of fixed poles. Therefore, no more expansion of their clamped-energy-knots takes place. So, those electrons don't intrude further their resting surface even to the extent of size (mass) of an electron, resulting to maintain the diamond state of their atom. Thus, the diamond-state carbon atom shows solid behavior at maximum extent. Therefore, the ground point of diamond-state carbon atom is at sufficiently below ground point of lonsdaleite-state carbon atom, which is a bit below to ground surface. In this context, lonsdaleite-state carbon atom is in less expansion of clamped energy knots to filled and unfilled states forming its lattice as compared to ones in diamond state carbon atom. In Figure 4, sketches of different entities are drawn in estimation to show less 
and more expansion of clamped energy knots to filled and unfilled states of carbon state atoms.
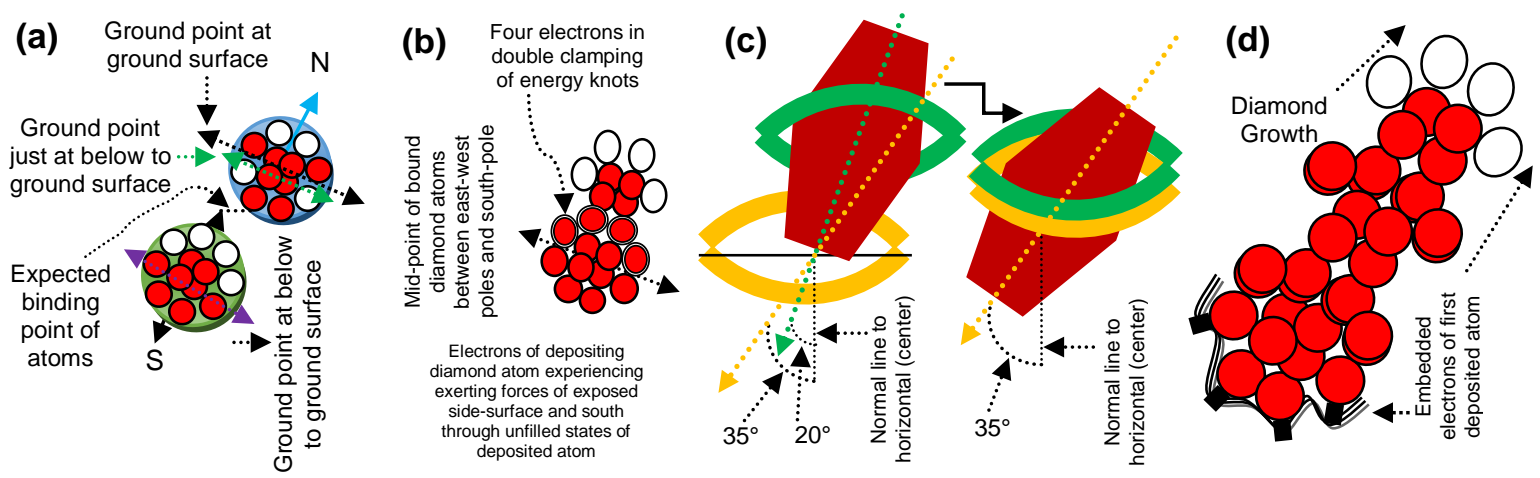

Figure 4: (a) ground points of lonsdaleite- and diamond-state carbon atoms along with the expected binding point of two diamond-state carbon atoms, (b) depositing diamond-state carbon atom when four electrons of outer ring undertake double clamping of energy knots under experiencing the forces of side-surfaces and south-poles where they rightly located above the four unfilled states of deposited diamond-state carbon atom, (c) orientation of certain electron of lonsdaleite-state carbon atom, prior to conversion and orientation, when it undertakes conversion into diamond-state carbon atom where clamping another energy knot belonging to certain unfilled state of deposited diamond-state carbon atom and (d) growth of diamond is south to ground; red colored circles indicate filled states, white colored circles indicate unfilled states and red colored double-circles indicate electrons of double clamping of energy knots.

The ground point of lonsdaleite-state carbon atom is just below the ground surface, because it is underneath the ground point of graphitic carbon atom. In diamond-state carbon atom, electrons are in their maximum gravitation behavior in the region where expansion of clamped energy knots also comes to the maximum extent. The resulting energy against the work done of electrons in diamond-state carbon atom dissipates, enabling the expansion of clamped energy knots to the maximum extent. Electrons of lonsdaleite-state carbon atom exert orientational force at lower degree angle from the normal line of their centre, resulting in lower amount of potential energy. Hence, the clamping energy knots are in lesser expansion.

On the transfer of left two electrons to downward-side unfilled states, lonsdaleitestate carbon atom is converted to the diamond-state carbon atom. Now, electrons of that converted diamond-state carbon atom also undertake the same level of expansion in terms of clamped energy knots as in the case of targeted (deposited) diamond-state carbon atom. On the other hand, depositing diamond atom on deposited diamond atom, a controlled expansion in clamped energy knots to their electrons should be anticipated as experiencing orientationally-controlled exerting 
forces (in surface-format and grounded-format) for relevant poles. This results in undertaking of targeted electrons (of depositing diamond-state carbon atom) for another clamping of energy knot through targeted unfilled states (of deposited diamond-state carbon atom) where they experienced the exerting force of relevant poles while arriving rightly over them (targeted unfilled states). Each electron of filled states belonging to outer ring of depositing diamond-state carbon atom undertakes another clamp of energy knot of unfilled states belonging to outer ring of deposited diamond-state carbon atom, resulting in their binding, as shown in Figure 4 (b). On binding diamond-state atoms, their combined filled and unfilled states, along with zeroth rings, adjust and compensate both expansion and contraction behaviors by constructing new binding point for the following depositing diamond-state carbon atom.

Lonsdaleite-state carbon atom undertakes less expansion of energy knots clamping electrons and vacant sites as compared to the diamond-state carbon atom. Therefore, a lonsdaleite-state carbon atom is more related to the recovery state of an atom where orientation of exerting force to each electron clamped by energy knot becomes $\sim 20^{\circ}$ angle from the normal line drawn from its centre $\left(270^{\circ}+20^{\circ}=290^{\circ}\right)$. Hence, in diamond-state carbon atom, expansion of clamped energy knot to electron takes place under the exertion of relevant poles forces at $\sim 35^{\circ}$ angle from the normal line drawn from its center $\left(270^{\circ}+35^{\circ}=305^{\circ}\right)$. The angles of exerting forces of relevant poles of electrons of lonsdaleite-state carbon atom and diamond state carbon atom from their normal line drawn at the center are shown in Figure 4 (c). The situation where electron of diamond-state when undertakes double clamping of energy knot to bind another diamond-state carbon atom is also shown, separately at right-side.

Overall growth behavior of diamond-state carbon atoms is shown in Figure 4 (d). The binding of diamond-state carbon atoms remained in-progress under the same mechanism on converting from gas-state carbon atoms. Here, diamond-state carbon atoms adjust and compensate contraction and expansion of their energy knots nets (and clamping energy knots to their electrons). This adjustment of nets is taken place each time of depositing (binding) a new diamond-state carbon atom to already deposited diamond-state carbon atom. Therefore, in diamond binding, growth behavior is from south to ground where binding point of the atoms remains between surface-format and grounded-format. Embedded electrons under suitable 
mechanism of the first deposited diamond-state carbon atom is also shown in Figure 4 (d), where they direct themselves ground to south under the maximum expansion of clamped energy knots. At that instant, electrons of depositing diamond atom remain detained in their clamped energy knots. So, when the binding of third diamond-state carbon atom is in process, those electrons of second deposited atom are being detained by the unfilled states of first deposited atom to undertake the already clamped energy knot to each of them firmly and precisely. A double clamping to electrons becomes apprehend on adjusting the expansion-contraction of the energy knots nets of two atoms. This came into force prior to bind with third diamond-state carbon atom. This can be referred to nucleation stage of diamond. Depositing the third diamond-state carbon atom, by locating a new point of binding with respect to already bound two deposited diamond-state carbon atoms, starts the growth process of diamond as shown in Figure 4 (d).

When the depositing diamond-state carbon atom reaches precisely over the deposited diamond-state carbon atom, two inside electrons of outer ring undertake exertion of force of the pole of outer-side (left-side electron west-pole and right-side electron east-pole) equal to the exertion of force of the south-pole where exertion of the force along the north-pole becomes negligible. On undertaking another clamp of energy knot (belonging to upward-side of deposited atom) by those two electrons (belonging to downward-side of depositing atom), their left two outer-side electrons also come into the precision to undertake another clamp of left two energy knots of outer-sides as shown in Figure 5. So, the mechanism of undertaking double clamp of energy knot by each electron is by the inner two electrons, following by the left two electrons for both quadrants of the south-pole of binding diamond-state carbon atom. Therefore, exertion of one-pole force to outer-side of the electron and one-pole force to tip-side remains diligent to control its position at instant of clamping another energy knot. The clamping of (another) energy knot is for the half-length to that electron when reached inside to its hollow region by undertaking the frictional (nonconservative) forces of various sections of infinitesimal displacements. Only the force exerts along one-pole having surface-format (exposed side-surface) and, similarly, only the force exerts along south-pole having grounded-format to undertake double clamping of energy knot. Both energy knots clamped by each electron expanded under disappeared exertion of one-pole force in surface-format and north-pole force in space-format are shown in Figure 5. In depositing diamond-state carbon atom, 
inner two electrons touch in-front electrons of zeroth ring, thus, further increasing their potential energy through infinitesimal displacements where orientationallycontrolled exerting forces result into another clamp of energy knot.
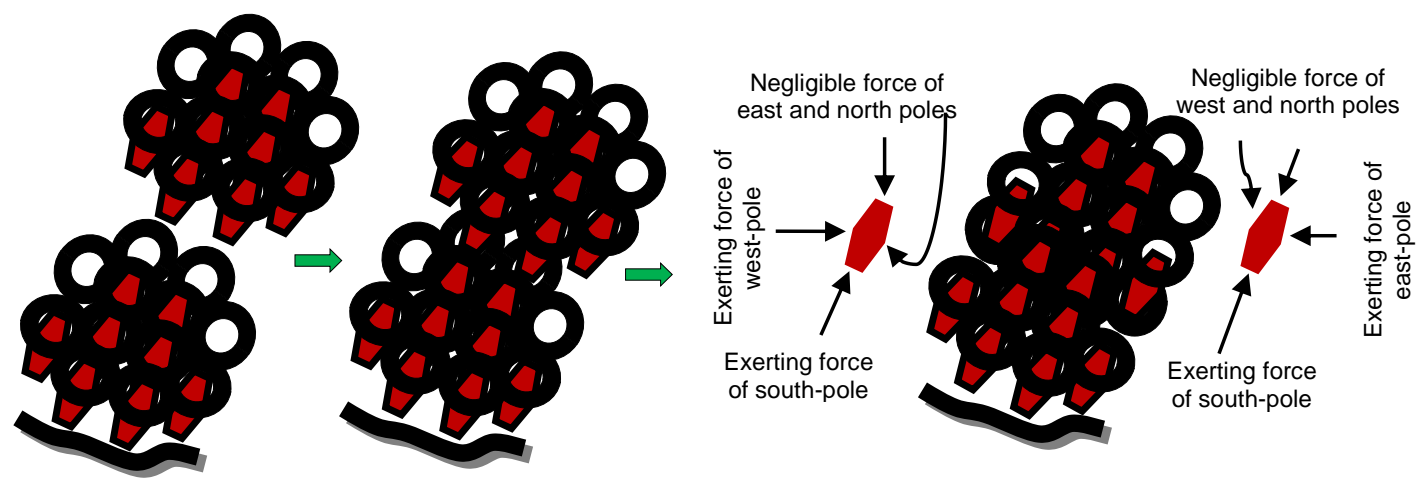

Figure 5: Binding of depositing diamond-state carbon atom to the rooted diamond-state carbon atom along with exertion of forces to the exposed sides of left-side electron and right-side electron

The mechanism of binding lonsdaleite-state atoms is identical to that of the case of binding diamond-state carbon atoms. However, only two oriented $\left(\sim 290^{\circ}\right)$ electrons of lonsdaleite-state atom clamp another clamping of energy knot belonging to deposited lonsdaleite-state carbon atom. In this manner, one atom experiences the force in grounded-format while the other atom in surface-format locating a new joint ground point. Therefore, binding in lonsdaleite-state carbon atoms is ground to just-south, but growth behavior is just-south to ground. The involved characteristic energy to convert gas-state carbon atom into lonsdaleite-state carbon atom is in the same shape as for the diamond-state carbon atom, but in fewer amounts. This is because of transferring of only two electrons along south-pole, at each left-side and right-side.

The ground point of graphene-state atom doesn't lie at ground surface but lies just above ground surface. Therefore, carbon atoms of graphene-state undertake contraction of clamping energy knots under exertion of force of electrons, where the levitation behavior is at pronounced level. Binding of graphene-state carbon atoms includes exerting forces in surface- and space-formats, where binding of atoms is ground to just-north. But, the growth of graphene is just-north to ground. This is the reason why graphene structure is based to only few layers as it is challenging to maintain exerting-forces for further elevation. Therefore, in evolution of graphene structure, the binding mechanism of atoms is opposite to the one as for diamond.

One more physical behavior of carbon atoms is resulted when layers of three different-states carbon atoms bind in repetitive manner, i.e., gas, graphite and 
lonsdaleite-state carbon atoms bind in successive manner. Exerting forces of relevant poles of electrons (belonging to different-state carbon atoms ordered as just described) are kept engaged by their involved (gained) potential energies, resulting into the binding of atoms of layers to evolve structure of glassy carbon. Layers of gas and graphite state atoms bind under the joint application of exerting forces in grounded- and surface-format, where involved orientationally-controlled potential energies of paired-electrons (of gas state carbon atoms) undertake double clamping of energy knots of paired unfilled states (of graphitic carbon atoms) by directing from their rear side. Gas-state carbon atoms attempt forcefully the gravitation behavior under increased potential energy of their electrons. Layers of lonsdaleite-state carbon atoms and graphite-state carbon atoms bind under the joint application of exerting forces in space-format and surface-format, where involved orientationallycontrolled potential energies of paired-electrons (of lonsdaleite state carbon atoms) undertake double clamping of energy knots of paired unfilled states (of graphitic carbon atoms) by directing from their front side. Lonsdaleite-state carbon atoms attempt forcefully levitation behavior under decreased potential energy of their electrons. Layers of Ionsdaleite-state carbon atoms and gas-state carbon atoms provide compensation in terms of expansion-contraction of bound layers of atoms as shown in Figure 6.

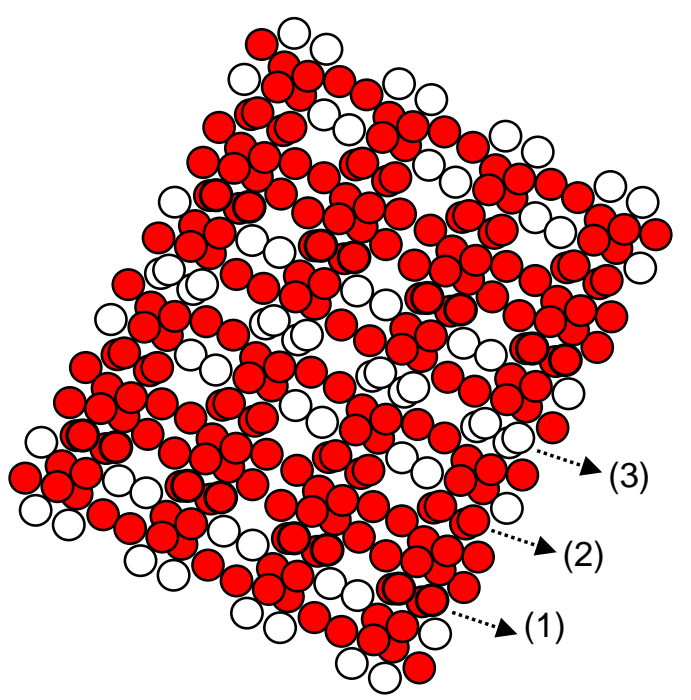

Figure 6: Structure evolution of glassy carbon where tri-layers of gas-, graphite- and lonsdaleitestate carbon atoms respectively bind in the successive manner; (1) paired electrons of each atom (belonging to gas-state carbon atoms layer) undertake double clamping of paired energy knots of each atom (belonging to graphitic carbon atoms layer) by entering from the rear sides, (2) paired electrons of each atom (belonging to lonsdaleite-state carbon atoms layer) undertake double clamping of paired energy knots (belonging to graphitic carbon atoms layer) by entering from the front 
sides and (3) layers of gas- and lonsdaleite-state carbon atoms provide compensation in binding their layers to the layer of graphitic carbon atoms

In carbon, electrons of outer ring transfer to nearby unfilled states, to originate different state behaviors of their atom. Transferring electrons of filled states to the unfilled ones in the carbon atom involves the non-conserved energy through which non-conservative forces engage. However, wherever the conservative forces involve addressing the dynamics of electrons of certain-natured atoms, they engage the conserved energy as well. As in the case of neutral-state silicon atom, where a filled state electron is transferred to nearby unfilled state under the exertion of conservative force where engaging the conserved energy also [1]. This indicates that atomic radius in different elements along with electronic structure of the atom is the core to elucidate what sort of the force-energy behavior is being considered by that atom.

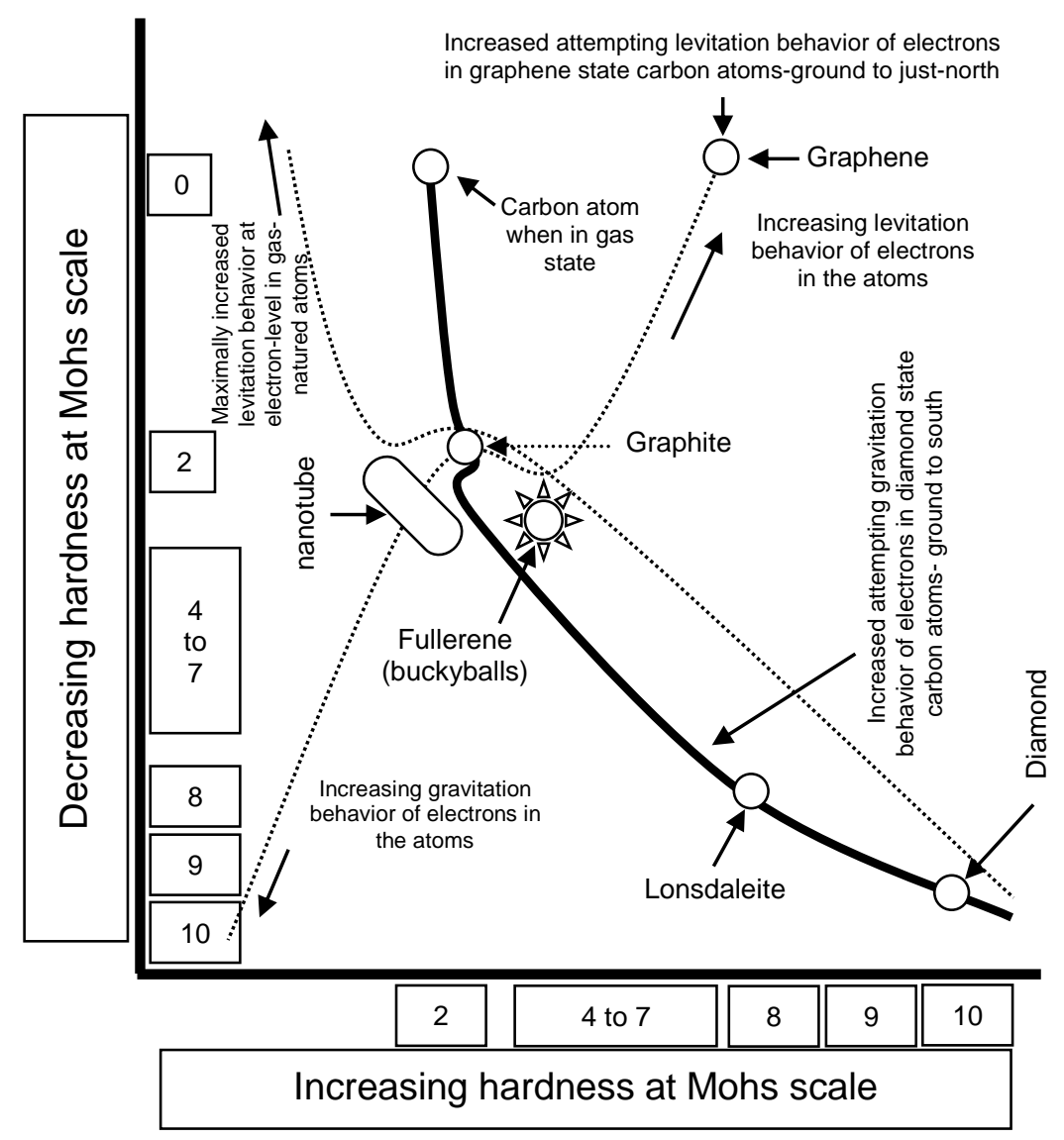

Figure 7: A sketch of approximately estimated hardness (at Mohs scale) of nanoscale structure in different states carbon atoms versus exerting levitational-gravitational force at electron-level

Hardness at Mohs scale for different structures of different states carbon atoms at nanoscale is sketched in Figure 7. No value of hardness is counted when undertaking the gas-state carbon atoms as they do not evolve structure at any scale. 
The hardness scale is related to the binding of different states carbon atoms, where involved non-conserved energies due to electron-dynamics engage their nonconservative forces, which is different for each established state of structure evolution of carbon atoms as noted above. In Raman spectroscopy, different values of wave number printed against energy signals of graphite structure and other structures of carbon (at nanoscale) reveal different nature of propagating photons through different inter-state electron gaps (in their different state carbon atoms), which is also validated by the energy loss spectroscopy [5].

\subsection{Conclusions}

Two units of energy, forming trajectory like shape of parabola, enable transferring of electrons from left-right sides to north-south poles of certain-state carbon atom while maintaining the equilibrium state, where exerting forces in respective format for relevant poles of those electrons work neutral. A carbon atom, where two electrons of outer ring occupy sites along the north-pole (at both left- and right-sides) and remaining two electrons of outer ring occupy sites just below the line of east-west poles, it is related to gas state. In the carbon atom, when two electrons of outer ring retain positions in the states available at just above the line of east-west poles and two electrons of outer ring retain positions in the states available at just below to that line, it is related to graphite state. A carbon atom where all the electrons of outer ring retain positions in the states at below east-west poles, it is related to diamond state.

When structure of graphitic carbon atoms evolves two dimensionally, it is under the application of attained dynamics only where difference in opposite pole forces in suitably amalgamated atoms regulate the structure. Forces exert under the difference of poles of amalgamated atoms. If they do not restrict them to bind under the engaged energies, at least, they also do not keep them from separating. So, atoms remain amalgamated (bound) under their appreciably attained dynamics only.

In bindings of graphite, nanotube and fullerene state carbon atoms, the involved typical energy shape-like parabola engages the neutral behavior of exerting forces to relevant poles of transferring electrons, which mainly fall in the surface-format. In the case where binding of graphitic carbon atoms is under the execution of electrondynamics, the evolution of structure is one dimensional. Carbon atoms in nanotubestate evolve two-dimensional structure, where energy involved for electrons in 
opposite quadrants of each atom engage neutral behavior of exerting forces to their relevant poles. Carbon atoms in fullerene-state evolve four-dimensional structure.

In bindings of diamond-, lonsdaleite- and graphene-state carbon atoms, the gained energy of electrons, undertaking double clamping of energy knots, is triggered by the orientationally-controlled exerting forces to their relevant poles. Bindings of diamond-state carbon atoms are ground to south, but growth is south to ground where potential energies of orienting electrons engage the controlled behavior of exerting forces to their relevant poles. So, it is a tetra-electrons ground to south topological structure. Bindings of lonsdaleite-state carbon atoms are ground to just-south, so it is a bi-electrons ground to just-south topological structure. Bindings of graphene-state carbon atoms follow opposite mechanism to that of the diamondstate carbon atoms. Here, potential energy of the orienting electrons engages the controlled behavior of exerting forces to their relevant poles both in the space- and surface-formats instead of the surface- and grounded-formats. So, it is a tetraelectrons ground to just-north topological structure.

Repeated sequence of tri-layers (gas-, graphite- and lonsdaleite-state carbon atoms) evolves structure of glassy carbon. In the structure evolution of glassy carbon, the electrons undertaking double clamping of the energy knots are entered from the rear side (bottom) in the case of layer of gas-state carbon atoms and from the front side (top) in the case of layer of lonsdaleite-state carbon atoms. Binding atoms of each layer of lonsdaleite-state to atoms of each layer of graphite-state is under attempting forcefully levitation behavior of electrons. This is because of decreased (lost) potential energy of electrons in each lonsdaleite-state carbon atom. So, the engagement of orientationally-controlled exerting forces of relevant poles to paired-electrons is from the front side and to undertake another clamping of energy knot. Binding atoms of each layer of gas-state to atoms of each layer of graphitestate is because of the forcefully gravitation behavior of electrons, where, because of their increased (gained) potential energy, they result into undertake another clamping of energy knots. Here, the engagement of orientationally-controlled exerting forces of relevant poles to paired-electrons is from the rear side.

The neutral forces at electron-levels in the formation of structures of graphite-, nanotube- and fullerene-state carbon atoms. They involve non-conserved energy to execute partially non-confined partially confined inter-state electron-dynamics. On the other hand, for structure evolution in diamond-, lonsdaleite- and graphene-state 
carbon atoms, non-confined inter-state electron-dynamics are executed where the involvement of non-conserved energies engage the frictional forces. The same is the case for structure evolution in glassy carbon.

Force and energy work inter-changeably; when force is involved, energy is engaged, and vice versa. The matter remains as the intermediate component identifying the functioning of force and energy. To originate the different physical behavior of each state carbon atom, non-conserved energies involve engaging nonconservative forces. The involved energy in developing structures of surface-format is non-conserved because it is a sub-part of conserved (discrete) energy of a unitphoton. However, for structure evolutions of diamond, lonsdaleite, graphene and glassy carbon, a transitional potential energy of electrons involve engaging orientationally-controlled exerting forces when double clamping of energy knots is undertaken by them. Each state carbon atom elaborates its own science and, so, in binding of different states carbon atoms. So, the carbon atom enables us to understand the nature of electron-dynamics in atoms of different class of elements along with originating new physical behaviors of atoms.

These investigations open new areas of research on different lines as compared to the existing ones. These investigations enable one to understand different phenomena related to optics and photonics, certain force-energy behaviors of atoms of different elements, designing of new materials and light-matter interactions.

\section{References:}

[1] M. Ali, Revealing the Phenomena of Heat and Photon Energy on Dealing Matter at Atomic level. https://www.preprints.org/manuscript/201701.0028/v10

[2] M. Ali, Why Atoms of Some Elements are in Gas State and Some in Solid State, but Carbon Works on Either Side (2018). https://www.researchgate.net/publication/323723379

[3] M. Ali, Structure evolution in atoms of solid-state dealing electron transitions under confined inter-state electron-dynamics. http://arxiv.org/abs/1611.01255v16

[4] M. Ali, Atoms of electron transition deform or elongate but do not ionize while inert gas atoms split under photonic current instead of electric. http://arxiv.org/abs/1611.05392v15 
[5] M. Ali, I -N. Lin, Phase transitions and critical phenomena of tiny grains carbon films synthesized in microwave-based vapor deposition system. Surf. Interface Anal. 2018;1-11. https://doi.org/10.1002/sia.6593

[6] M. Ali, M. Ürgen, Switching dynamics of morphology-structure in chemically deposited carbon films -A new insight, Carbon 122 (2017) 653-663.

[7] M. Ali, M. Ürgen, Deposition Chamber Pressure on the Morphology-Structure of Carbon Films (2018). https://arxiv.org/abs/1802.00730v7

[8] M. Ali, M. Ürgen, Simultaneous growth of diamond and nanostructured graphite thin films by hot filament chemical vapor deposition, Solid State Sci. 14 (2012) 150-154.

[9] S. Link, M. A. El-Sayed, Shape and size dependence of radiative, nonradiative and photothermal properties of gold nanocrystals, Int. Rev. Phys. Chem. 19 (2000) 409- 453.

[10] S. C. Glotzer, M. J. Solomon, Anisotropy of building blocks and their assembly into complex structures, Nature Mater. 6 (2007) 557-562.

[11] M. Ali, I -N. Lin, The effect of the Electronic Structure, Phase Transition, and Localized Dynamics of Atoms in the formation of Tiny Particles of Gold, http://arXiv.org/abs/1604.07144v10

[12] M. Ali, I $-\mathrm{N}$. Lin, Development of gold particles at varying precursor concentration, http://arxiv.org/abs/1604.07508v12

[13] M. Ali, I -N. Lin, C. -J. Yeh, Tapping Opportunity of Tiny-Shaped Particles and Role of Precursor in Developing Shaped Particles, NANO 13 (7) (2018) 1850073 (16 pages).

[14] M. Ali, I -N. Lin, Controlling morphology-structure of particles at different pulse rate, polarity and effect of photons on structure. http://arxiv.org/abs/1605.04408v12

[15] M. Ali, I -N. Lin, Formation of tiny particles and their extended shapes: origin of physics and chemistry of materials, Appl. Nanosci. 9 (2019) https://doi.org/10.1007/s13204-018-0937-z. http://arxiv.org/abs/1605.09123v16

[16] M. Ali, The study of tiny-shaped particles developing mono-layer dealing localized gravity at solution surface. http://arxiv.org/abs/1609.08047v16

[17] M. Ali, Nanoparticles-Photons: Effective or Defective Nanomedicine, J. Nanomed. Res. 5 (2018) 241-243. 
[18] M. Ali, I -N. Lin, C. -J. Yeh, Predictor Packing in Developing Unprecedented Shaped Colloidal Particles, NANO 13 (9) (2018) 1850109 (15 pages).

[19] M. Ali, E. Hamzah, M. R. M. Toff. Hard Coating is Because of Oppositely Worked Force-Energy Behaviors of Atoms. https://www.preprints.org/manuscript/201802.0040/v8

[20] M. Ali, I -N. Lin. Precise Structural Identification of High Aspect Ratios Gold Particles Developed by Unprecedented Machinic Approach. https://www.researchgate.net/publication/329066950

[21] Y. Gao, et al., Ultrahard carbon film from epitaxial two-layer graphene, Nature Nanotechnol. 13 (2018) 133-138.

[22] J. -B. Wu, M. L. Lin, X. Cong, H. N. Liu, P. H. Tan, Raman spectroscopy of graphene-based materials and its applications in related devices, Chem. Soc. Rev. 47 (2018) 1822-1873

[23] R. Shoukat, M. I. Khan, Synthesis of vertically aligned carbon nanofibers using inductively coupled plasma-enhanced chemical vapor deposition, Electr. Eng., 100 (2018) 997-1002

[24] M. S. Cao, et al., Graphene nanohybrids: excellent electromagnetic properties for the absorbing and shielding of electromagnetic waves, J. Mater. Chem. C, 6 (2018) 4586-4602.

[25] L. Fu, et al., A glassy carbon electrode modified with N-doped carbon dots for improved detection of hydrogen peroxide and paracetamol, Microchimica Acta 185 (2018) 87.

[26] D. Y. Hu, J. X. Hu, H. L. Jiang, J. Xu, A highly effective energy mitigation system combining carbon nanotube and buckyballs, Eur. Phys. J.-Spec. Top. 127 (2018) 155-166.

[27] C. P. Hsu, et al., Buckball-, carbon nanotube-, graphite-, and grapheneenhanced dehydrogenation of lithium, Chem. Commun. 49 (2013) 8845-8847.

[28] C. Y. Chen, et al., Laser writing of coherent colour centres in diamond, Nature Photonics 11 (2017) 77-80. 
[29] Z. Liu, et al., Graphite-graphene architecture stabilizing ultrafine $\mathrm{Co}_{3} \mathrm{O}_{4}$ nanoparticles for superior oxygen evolution, Carbon 140 (2018) 17-23.

[30] A. taylor, et al., Precursor gas composition optimisation for large area boron doped nano-crystalline diamond growth by MW-LA-PECVD, Carbon 128 (2018) 164-171.

[31] X. Cheng, W. J. Zong, Anisotropic evolution of damaged carbons of a mechanically polished diamond surface in low-temperature annealing, Diam. Relat. Mater. 90 (2018) 7-17.

[32] M. Maruyama, S. Okada, Geometric and electronic structure of a twodimensional covalent network of $\mathrm{sp}^{2}$ and $\mathrm{sp}^{3}$ carbon atoms, Diam. Relat. Mater. 81 (2018) 103-107.

[33] A. Narjabadifam, F. Vakili-Tahami, M. Zehsaz, Elastic and failure properties of carbon nanocones using molecular dynamics simulation, Fuller. Nanotub. Carbon Nanostruct. 26 (2018) 777-789. 